\section{Biochemistry has outgrown its traditional boundaries}

SIR - Your Editorial "What's in a name?" (Nature 442, 486; 2006) makes the case for the importance of chemistry to current 'molecular sciences' but contains the unfortunate statement that biochemistry is "associated primarily with the study of enzyme kinetics". This is a narrow and dated view of the subject.

In fact, the revolution in modern biology has been underpinned by the application of traditional biochemistry to fields as diverse as genetics, cell biology and ecology. To acknowledge the importance of chemistry to biology but to regard biochemistry as little more than the study of enzyme kinetics displays an ignorance both of the courses that make up a modern degree in biochemistry and of the content of biochemistry journals.

What is in a name? Many traditionally trained biochemists would happily describe themselves as molecular or cellular biologists. The boundaries in science have all but disappeared, and a good thing too.

However, if we are to debate traditional labels, please don't take the richly diverse subject of biochemistry and define it by a single discipline.

Chris Kirk

The Biochemical Society, Eagle House, 16 Procter Street, London WCIV 6NX, UK

\section{Videos have starring role to play in protocol sharing}

SIR - Who has never struggled with a new protocol? The electronic initiatives to share and discuss protocols, as reported in the News story "Online methods share insider tricks ${ }^{\circ}$ (Nature 441, 678; 2006) and in Correspondence ("Wiki and other ways to share learning online" Nature 442, 744; 2006), are to be welcomed. Unfortunately, even a detailed written protocol cannot have the same effect as sitting beside someone who is doing it.

Probably the most feasible approach is to publish movies describing the methods, a service already offered by some publications and protocol websites, but which could become routine. Much more information on the essential steps of a new protocol, including audio commentary on the trickiest steps (from the position of the Petri dishes to the speed of dispensing), could be accessible using video format and published online with the paper. Such videos could transform the way in which methods are communicated.

Readers may object that they don't have time to set up Hollywood studios to publish a paper. But the effort required for filming a relevant procedure is outweighed by the fact that more precise description of methods greatly aids scientific understanding and facilitates future research. Furthermore, videos are valuable tools for teaching.

\section{Matias Pasquali}

Centre of Competence for Innovation in the

Agro-environmental Sector, University of Torino, and Department of Plant Pathology, 1991 Upper

Bufard Circle, University of Minnesota,

Saint Paul, Minnesota 55108-6030, USA

\section{Consistency tests establish empirical generalizations}

SIR - Your Editorial "Let's replicate" and News Feature "The trouble with replication" (Nature 442, 330 and 344-347; 2006)

address the problem of how to obtain reliable knowledge in science. These articles usefully point to difficulties in conducting successful replications, and note the change in social processes associated with resolving contested experimental results. Taken together, though, they risk misleading the reader about the process of replication in science.

Your Editorial acknowledges the diversity of the practice of replication in science, but uses a rather narrow definition of replication. Bywriting of "direct", "full" and "exact" replication, you seem to presuppose an ideal of literal replication - the idea that replication involves attempts to produce a 'carbon copy' of the original experiment. Of course, such an objective cannot be attained, if only because of the unavoidable changes in time, location and investigative personnel that replication brings with it.

Close replication is a more realistic aim in science than literal replication. A close replication uses the conditions and procedures of the initial study, perhaps correcting for its perceived inadequacies. It is a checking strategy that attempts to achieve similar results to the first study.

Close replication must, however, be distinguished from constructive replication. The latter process is undertaken to demonstrate the extent to which results hold across different experimental methods, treatments, populations and occasions. It is a triangulation strategy designed to reveal the extent to which the results identified by successful close replication can be generalized. Constructive replication is of vital importance to science because it is the main means by which empirical phenomena are detected. Your Editorial mentions the scientific aim of confirming and extending unpublished experimental conclusions, but in my view it errs in not stating that this is a form of replication.

To be sure, craft or tacit knowledge is often needed to establish the reliability associated with effectively carrying out experimental studies and successfully replicating their findings. Social studies of science have made clear that there is also an unavoidable social dimension to the successful practice of experimental science. However, it is the successful use of consistency tests in the practice of experimental replication that provides the evidential basis for its claims about empirical phenomena.

Brian D. Haig

Department of Psychology,

University of Canterbury,

Private Bag 4800, Christchurch,

New Zealand

\section{Chimp comparisons won't explain human evolution}

SIR - You state in your News story on genetic differences between humans and other species ( ${ }^{\circ} \mathrm{Mix}$ and match: the hunt for what makes us human" Nature 443, 8; 2006) that research is beginning to pin down genes that "evolved rapidly during the transition from chimps to people". No such transition occurred, of course, because chimpanzees are not human ancestors; they have been evolving for exactly the same amount of time.

A mutation is, in principle, just as likely to have occurred in the chimpanzee lineage as in the human lineage, during the time since their common ancestor lived. There is no way to discover from any comparison of the two species which is the case.

To determine the evolutionary history of a trait and its genetic basis, it is necessary to make phylogenetic reconstructions based on comparisons among many species. Chimp-human differences make good headlines, but tell us little about how human uniqueness evolved.

Robert Barton

Evolutionary Anthropology Research Group, Durham University, 43 Old Elvet, Durham DH13HN, UK

\section{Science prediction rate would be good in gambling}

SIR - I was surprised to read in Don Ihde's review of David Nye's Technology Matters: Questions to Live With (Nature 442, 984; 2006) that ${ }^{\alpha}$ of the 1,500 published predictions from scientists, inventors and sociologists [historian George Wise] surveyed, only a third were fulfilled". To be precise, I wonder at the use of the word "only". Anyone able to predict the outcome of a horse race one out of three times could make a handsome income. It seems an impressive success rate to me. Kenneth D. Pimple

Poynter Center for the Study of Ethics and American Institutions, Indiana University, Bloomington, Indiana 47405-3602, USA 